Research Article

\title{
Saving the Breast Saves the Lives of Breast Cancer Patients
}

\author{
Mohammad Esmaeil Akbari, Maryam Khayamzadeh, Hamid Reza Mirzaei, \\ Afshin Moradi $(\mathbb{D}$, Atieh Akbari $\mathbb{D}$, Farid Moradian, and Neda Khalili $(\mathbb{D}$
}

Cancer Research Center, Shahid Beheshti University of Medical Sciences, Tehran, Iran

Correspondence should be addressed to Atieh Akbari; akbari.atieh@yahoo.com

Received 23 October 2019; Revised 6 January 2020; Accepted 21 January 2020; Published 27 February 2020

Academic Editor: C. H. Yip

Copyright (c) 2020 Mohammad Esmaeil Akbari et al. This is an open access article distributed under the Creative Commons Attribution License, which permits unrestricted use, distribution, and reproduction in any medium, provided the original work is properly cited.

\begin{abstract}
Introduction. Surgery has been known as the procedure of choice for breast cancer management since 1700 years before Christ. Nowadays, breast-conserving surgery and mastectomy are performed in selected cases with specific clinical criteria. Here, we compare these two procedures for breast cancer patients with variable features in Cancer Research Center, Tehran, as a single institution experience. Methods. In this 25-year follow-up retrospective cohort study, we identified breast cancer patients who had undergone breast-conserving therapy or mastectomy. Disease-free survival and overall survival were evaluated using Kaplan-Meier survival analysis and the log-rank test between the two groups. A $p$ value less than 0.05 was considered statistically significant. Results. A total of 3358 breast cancer patients, including $61 \%$ breast-conserving therapy and $39 \%$ mastectomy cases were identified, with a mean follow-up time of 94 months. The overall survival and disease-free survival of all cases were significantly better in breast-conserved patients, particularly in early-stage breast cancer with favorable clinical, pathological, and biological features. Ten-year disease-free survival and overall survival in breast-conserving therapy and mastectomy cases were $74 \%, 88 \%$ and $58 \%, 80 \%$, respectively. Conclusion. Breast-conserving surgery and radiation therapy prove to be an appropriate treatment option for breast cancer patients in terms of overall survival and disease-free survival when indicated.
\end{abstract}

\section{Introduction}

Breast cancer, the most common malignancy in women worldwide, is a major public health concern. In 2018, 18.1 million new cases were diagnosed with breast cancer, and 9.6 million deaths occurred due to cancer. Also, the 5-year prevalence was estimated to be 43.8 million in the world [1]. Nowadays, management of breast cancer is largely dependent on surgery, radiation, and systemic therapy. Surgery has been introduced as the mainstay of treatment in breast cancer since approximately 1700 BC [2].

Galen, the Greek surgeon (129-210 AD), believed that breast cancer is a systemic disease and proposed that the accumulation of excess black bile accounts for pathogenesis [3]. Based on a very popular document from Avicenna (Canon), surgery has been well defined for an early-stage breast cancer and metastatic to the axilla [4].
All of the physicians, including Hippocratic surgeons (after the 4th century BC), Archigenes of Apamea (1st-2nd century AD), Galen (2nd century AD), Leonides of Alexandria (circa 2nd century AD), and Avicenna (c.980-June 1037) believed that surgery is effective when the cancer is limited to the breast and not locally advanced; they also recommended to avoid surgery when it is ulcerated or fixed to the axilla and helped the patients in such cases with medicament $[3,4]$.

Surgery has remained to be the most important procedure in the treatment of breast cancer since the ancient period. In the 1900s, William S Halsted introduced a novel surgical technique, known as radical mastectomy, after conducting a nonrandomized but controlled clinical trial which demonstrated improvement in the locoregional control of the disease. However, no changes were observed in terms of overall survival [5]. 
After this period, randomized clinical trials of breastconserving surgery (BCS) were pioneered in Milan by professor Veronesi [6-8] as the National Surgical Adjuvant Breast and Bowel Project (NSABP) chaired by Dr. Bernard Fisher. They defined the benefits and the techniques of this less morbid treatment approach as compared with the more popular radical mastectomy [9].

During the Fisher era, scientists challenged the mastectomy procedure and breast cancer was known as a systemic disease, which is probably controllable by less extensive surgery and by conserving the breast plus radiotherapy. It was confirmed by many trials that BCS in combination with radiation therapy is similar to modified radical mastectomy (MRM) for local/systemic control of breast cancer $[6,7,9-13]$. In all of these trials, not only was there no inferiority for BCS versus mastectomy regarding local recurrences, distant metastases, and overall survival but also many studies showed benefits for breast conservation therapy (BCT) over mastectomy. Besides, some trials demonstrated a slightly more favorable outcome in patients with positive nodes treated with BCS; however, the differences were not statistically significant [14-20].

In a pooled data from the National Cancer Institute (NCI), European Organization for Research and Treatment of Cancer (EORTC), and Institut Gustave Roussy (IGR) trials $[15,21]$, the odds ratio for overall survival (OS) at 10 years was more favorable in BCT compared with mastectomy. Also, improved survival was observed in BCT patients with positive axillary node over mastectomy cases who did not receive postmastectomy radiation. In another study, although no significant difference in OS was found, local recurrences were less in BCT versus mastectomy (5.9\% vs. $6.2 \%)[15]$.

Previous studies supported the concept that breast cancer is a systemic disease with local manifestations and radicalistic surgery does not statistically change overall survival; also, these studies confirmed that breast cancer surgery plus radiotherapy (RT) is not an inferior strategy compared with mastectomy.

Recently, some trials have confirmed the superiority of BCS with radiation therapy compared with mastectomy $[14,15,21]$.

In this 25-year follow-up study, we aimed to investigate the survival outcomes of female breast cancer patients undergoing BCT or mastectomy.

\section{Materials and Methods}

In this large, retrospective cohort study, we identified breast cancer patients who underwent BCT or mastectomy at the Cancer Research Center between 1991 and 2016. We included patients irrespective of their age, pathologic stage (IIV), primary tumor size, axillary status, and pathological and biological pattern with no evidence of gross multicentricity or diffuse noninvasive pathology and extensive microcalcification. All male breast cancer patients and those lost to follow-up were excluded from the study.

All of the BCS cases who had positive axillary lymph nodes and tumor size of more than $2 \mathrm{~cm}$ received additional chemotherapy (CT) and radiotherapy (RT), approximately $50 \mathrm{~Gy}$ with boost dose, after surgery. All the remaining cases who did not receive CT, however, had received RT as an integral part of BCT management.

Mastectomy cases were patients of any age or nodal status with/without neoadjuvant chemotherapy who had extended ductal carcinoma in situ (EDCIS) and also those who preferred mastectomy over BCS. Node-positive mastectomy cases also received CT and RT, whereas nodenegative total mastectomy cases with noninvasive breast cancer did not receive CT or RT.

This retrospective cohort study analyzed 3358 breast cancer cases in different stages with 25 years follow-up in two groups of BCS plus RT versus mastectomy cases. For the purpose of analysis, patients were divided into six age groups: $<30,30-39,40-49,50-59,60-69$, and $\geq 70$. The number of positive nodes was categorized as follows: $0,1-3$, $4-10$, and $>10$. Data for age at diagnosis, tumor size, pathology (invasive vs. non-invasive), stage of the disease, number of positive lymph nodes, Estrogen Receptor (ER) status, Progesterone Receptor (PR) status, and Her-2 status were extracted for all of the patients. OS was defined as the time from the date of diagnosis to the date of the last followup or death (due to any reason). Disease-free survival (DFS) was defined as the time from the date of diagnosis to the date of recurrence. Patient characteristics for the BCT or mastectomy cases were compared using two-sided $t$-tests and chi-square tests. The Kaplan-Meier analysis provided estimates of OS and DFS; statistical significance between BCT and mastectomy groups was accomplished with log-rank testing. All patients with noninvasive disease were excluded from the survival analysis, since noninvasive disease does not affect survival. Data analysis was performed using the SPSS software (version 22), and a $p$ value of less than 0.05 was considered as statistically significant in the analysis.

All procedures performed in our study were in accordance with the ethical standards of the institution and/or national research committee and with the 1964 Helsinki Declaration and its later amendments or comparable ethical standards. The ethical committee of Shahid Beheshti University of Medical Sciences approved this study, and written informed consent was obtained from the patients or from parents or legal guardians for minors or incapacitated adults.

\section{Results}

In this retrospective cohort study, a total of 3358 breast cancer patients who had referred to Cancer Research Center, Tehran, between 1991 and 2016 and had undergone BCT or mastectomy were analyzed. The mean follow-up time for the entire cohort was 94 months. Of the total cases, 2050 (61\%) underwent BCT. Only $2 \%$ and $5 \%$ of patients in BCS and mastectomy groups had stage 4 disease. The majority of patients in the BCT and mastectomy groups had invasive cancer (94\% and 96\%, respectively). The mean age of patients was 49.12 years $(S D=11.58)$ with no significant difference between the two groups. Patient and tumor characteristics for both groups are summarized in Table 1. The treatment groups were similar with respect to age. The 
TABLe 1: Characteristics of the two groups of patients.

\begin{tabular}{lccc}
\hline Subject & BCT $^{¥}$ & Mastectomy & $P$ value \\
\hline Number (\%) & $2050(61)$ & $1308(39)$ & \\
Mean age (year) & 48.95 & 49.47 & 0.25 \\
Stages & & & \\
I & $27.4 \%$ & $8.7 \%$ & \\
II & $49.1 \%$ & $40.8 \%$ & $<0.001$ \\
III & $21.6 \%$ & $45.7 \%$ & \\
IV & $1.8 \%$ & $4.8 \%$ & \\
Tumor size & & & \\
T1 & $39.6 \%$ & $18.2 \%$ & $<0.001$ \\
T2 & $55.3 \%$ & $59.2 \%$ & \\
T3 & $5.1 \%$ & $22.3 \%$ & \\
Number of positive nodes & & & \\
0 & $55.5 \%$ & $34.8 \%$ & \\
1-3 & $26.2 \%$ & $26.7 \%$ & $<0.001$ \\
$4-10$ & $13.6 \%$ & $26 \%$ & \\
$>10$ & $4.8 \%$ & $12.8 \%$ & \\
Pathology & & & \\
Noninvasive & $6.1 \%$ & $3.7 \%$ & $<0.002$ \\
Invasive & $93.9 \%$ & $96.3 \%$ & \\
Radiation therapy & $98.9 \%$ & $88.3 \%$ & $<0.001$ \\
ER+ & $74.6 \%$ & $66 \%$ & $<0.001$ \\
PR+ & $69.1 \%$ & $56.5 \%$ & $<0.001$ \\
Her-2+ & $19.9 \%$ & $30.9 \%$ & $<0.001$ \\
Post NACT & $8.8 \%$ & $13.8 \%$ & $<0.001$ \\
Chemotherapy & $93.4 \%$ & $97.9 \%$ & $<0.001$ \\
\hline Bren & &
\end{tabular}

${ }^{{ }^{*}}$ Breast conserving therapy. ${ }^{\circledR}$ Postneoadjuvant chemotherapy.

age distribution of patients is shown in Figure 1. As shown in Figure 2, the trend in the kind of surgery performed for breast cancer patients has changed over the past 20 years, with BCS increasing from $21 \%$ to $77 \%$.

Figure 3 demonstrates the 5- and 10-year overall survival in the two groups, which is significantly better in the BCT group; 5- and 10-year OS rates in BCS and mastectomy cases were $95 \%, 88 \%$, and $90 \%, 80 \%$, respectively. When the patients were further subdivided by the pathologic stage, the BCTgroup continued to show non-inferiority in terms of OS compared with the mastectomy group (Figure 4). In this study, a better DFS was also observed in the BCT group compared with the mastectomy group (Figure 5); regarding DFS, 5- and 10-year rates in BCT and mastectomy cases were $86 \%, 74 \%$, and $78 \%, 58 \%$, respectively.

Table 2 demonstrates the OS and DFS rates in the two groups, BCT and mastectomy, in this study and previous studies.

\section{Discussion}

This is a single institute experience with a mean follow-up of 94 months regarding the kind of surgery for breast cancer patients with different criteria. Historically, the clinical outcomes of BCS followed by radiation therapy and mastectomy are similar to each other, and even the overall survival is similar to that of lumpectomy cases without radiation therapy [10-14].

In line with the previous studies, our study showed that the overall survival was significantly better in BCS compared with mastectomy. After dividing the patients based on their

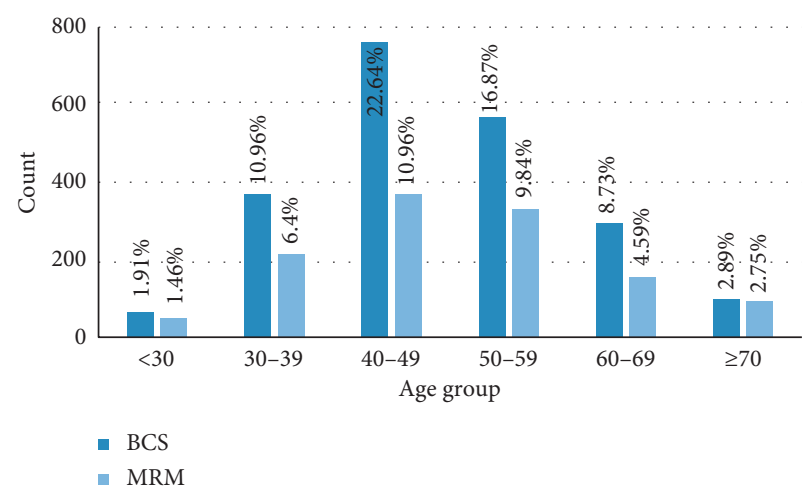

FIgURE 1: Age distribution of the patients.

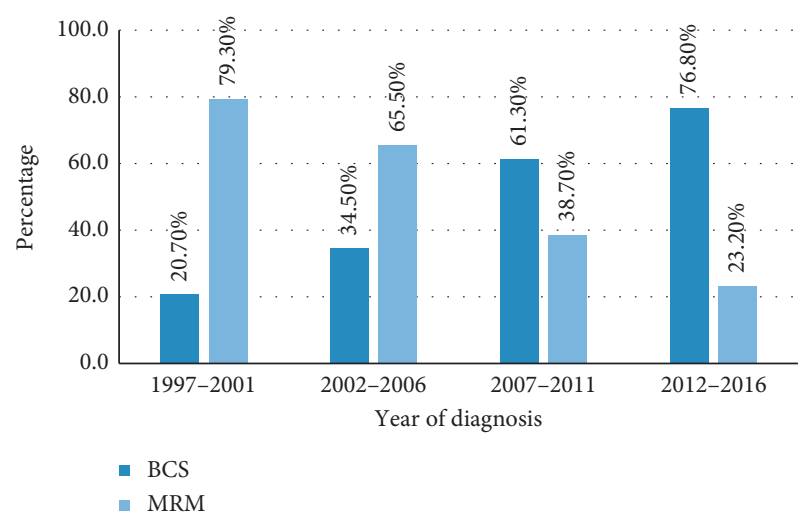

Figure 2: Variation trend based on the kind of surgery.

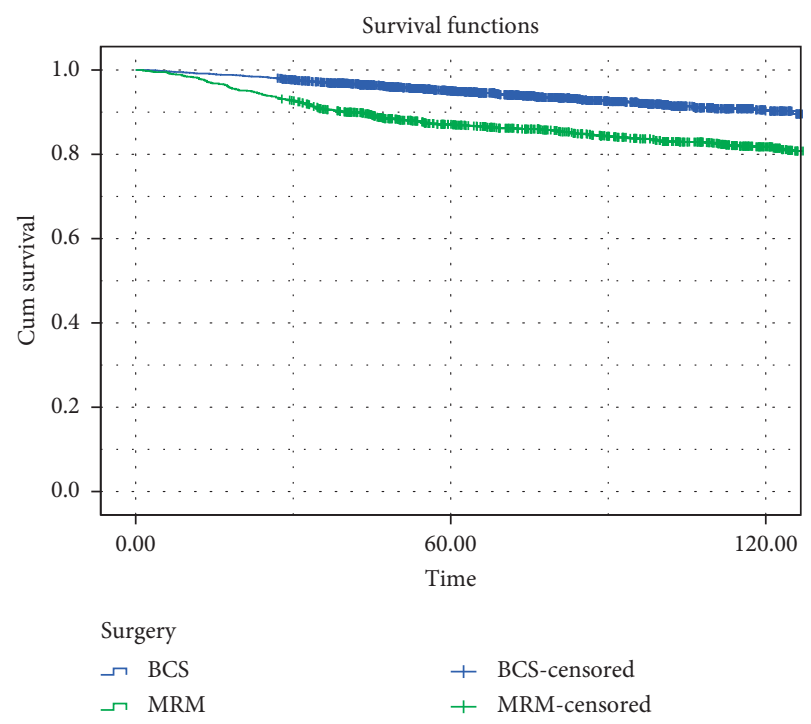

FIGURE 3: Five- and 10-year overall survival in all patients with BCS and mastectomy.

pathologic stage, similar results were observed; meaning that saving the breast saves the life of breast cancer patients with different stages, providing the standard management. Also, the DFS was significantly better in the BCT group as 


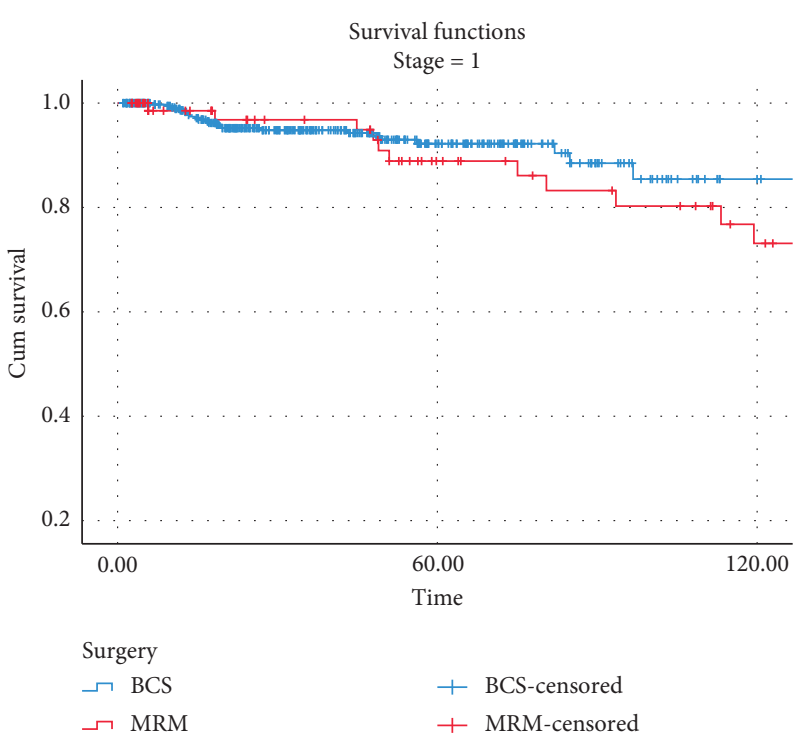

(a)

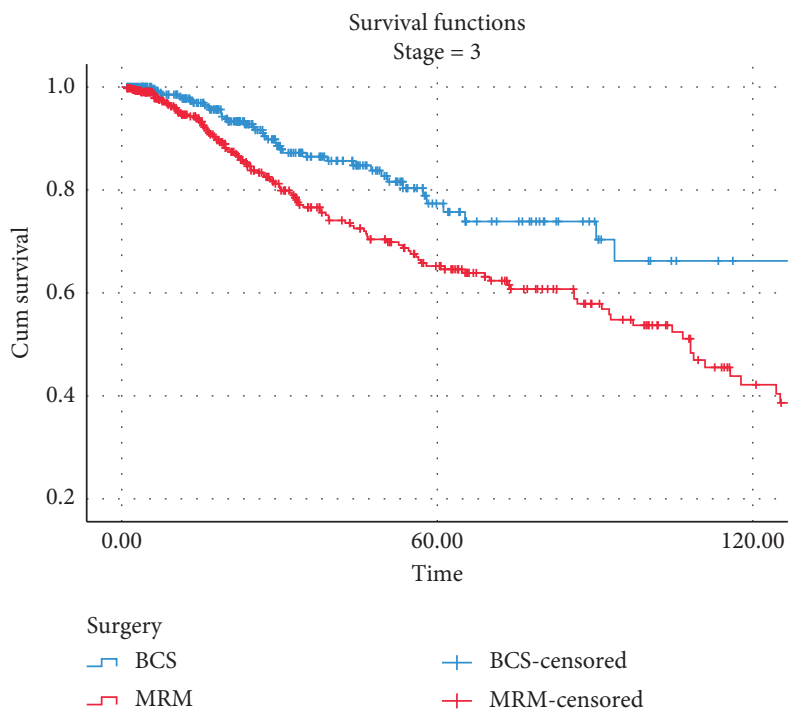

(c)

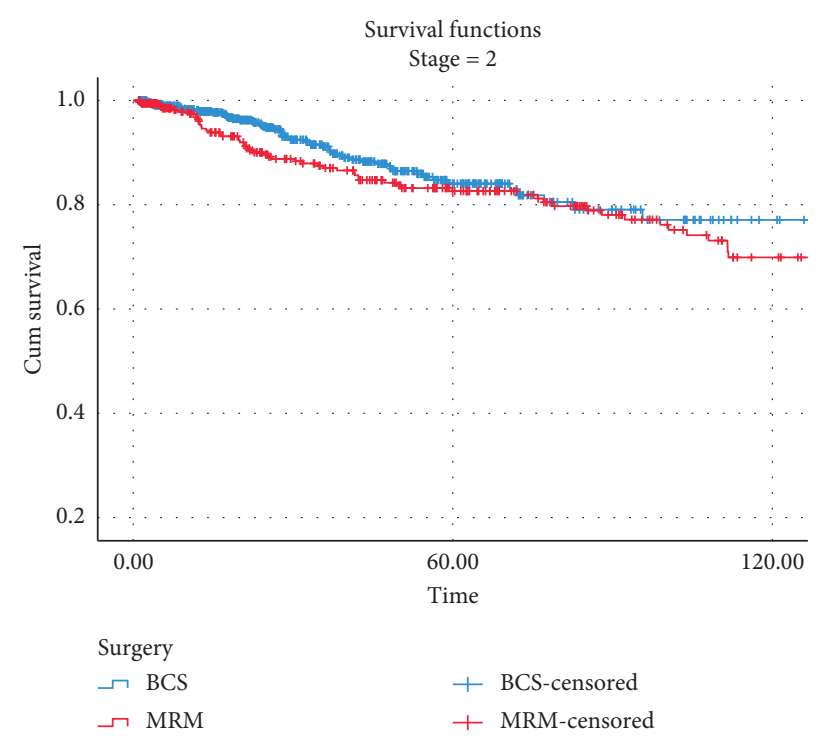

(b)

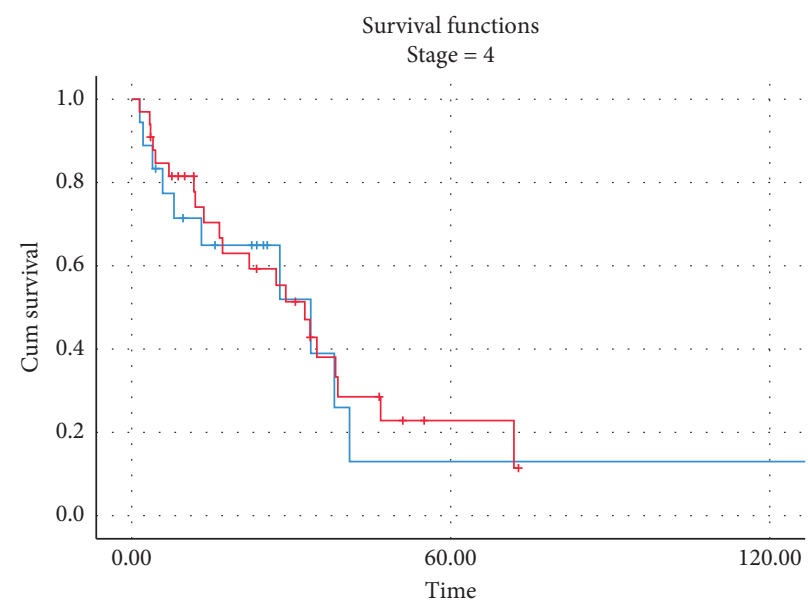

Surgery

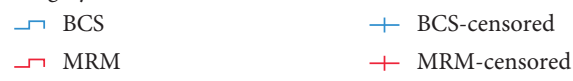

(d)

Figure 4: Overall survival based on the kind of surgery and stages. (a) Stage $1 P=0.02$. (b) Stage $2 P=0.012$. (c) Stage $3 P=0.04$. (d) Stage 4 $P=0.087$.

compared with the mastectomy cases, which shows that $\mathrm{BCT}$ has a significantly considerable systemic action that could be explained by the abscopal effect. Hence, we highly recommend breast-conserving treatment, particularly in earlystage breast cancer with better biological and pathological status.

In three randomized trials conducted in Italy, 349 mastectomy cases, 1006 cases with quadrantectomy + radiotherapy, 345 lumpectomies + radiotherapy cases, and 273 cases with quadrantectomy without radiotherapy were randomized, and the rate of local recurrences were $2.3 \%, 3.3 \%, 12.8 \%$, and $11.7 \%$, respectively. Although the mastectomy and quadrantectomy plus radiotherapy groups had a low incidence of recurrence compared with the other two groups, the statistical significance of the differences was negligible or did not exist at all [13]. In a population-based study in the Netherlands and Denmark, it was shown that BCS plus RT improved the OS in all cases compared with mastectomy, even in T1N0 cases; and also, the 10-year distant metastasis-free survival was significantly better in BCS [22-24].

Based on the current literature, not only clinical criteria such as tumor size and nodal status affects overall survival but also biological and pathological criteria are considered as important factors with a major impact on overall survival. In this regard, some standard managements have been established based on these new findings [25].

One of the most important reasons for improved OS regarding the kind of surgery is theoretically believed to be due to the effect on special aspects such as Quality of Life 


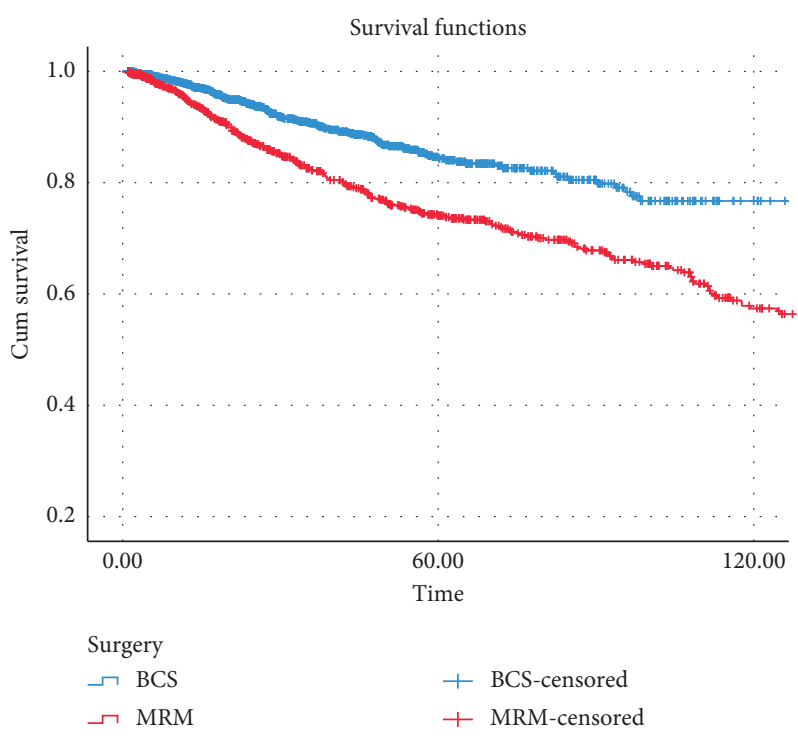

FIGURE 5: Five- and 10-year disease-free survival in all patients with BCS and mastectomy.

TABLE 2: Results of 5-year OS and DFS in prospective randomized trials comparing breast conservative surgery and radiotherapy (BCT) with mastectomy.

\begin{tabular}{|c|c|c|c|c|c|}
\hline \multirow{2}{*}{ Trial } & \multirow{2}{*}{$\begin{array}{l}\text { Endpoint } \\
\text { (years) }\end{array}$} & \multicolumn{2}{|c|}{ Overall Survival (\%) } & \multicolumn{2}{|c|}{ Disease-free Survival (\%) } \\
\hline & & BCS \& RT & Mastectomy & BCS and RT & Mastectomy \\
\hline NCI milan [15] & 18 & 65 & 65 & & N/A \\
\hline Institut Gustav Roussy [15] & 15 & 73 & 65 & & N/A \\
\hline NSABP B-06 [15] & 12 & 63 & 59 & 50 & 49 \\
\hline NCI USA [15] & 10 & 77 & 75 & 72 & 69 \\
\hline EORTC [15] & 8 & 54 & 61 & & N/A \\
\hline Danish breast cancer group [15] & 6 & 79 & 82 & 70 & 66 \\
\hline Akbari et al. [14] & 13 & 86 & 78 & & N/A \\
\hline Akbari et al. (Current study) & 25 & 95 & 88 & 86 & 76 \\
\hline
\end{tabular}

OS: Overall survival, DFS: disease-free survival, N/A: not applicable, BCS and RT: breast-conserving surgery and radiotherapy.

(QOL), with psychosocial effects on patients who have preserved their breast.

Several previous studies have confirmed psychosocial and spiritual relationship with improvement in QOL and OS during the management of breast cancer patients. According to these research hypotheses, social support and personality traits have an impact on the OS of patients with breast cancer. Social support helps women suffering from breast cancer experience better understanding and coping strategies. In all of these studies, the patients' trust, forgiveness, social support, personality, and other psychosocial and spiritual elements affect QOL and survival in breast cancer patients [26-28].

Studies have shown that patients who preserve their breast have higher self-esteem and disease acceptance compared with patients who have undergone mastectomies [29].

The second important reason for improved OS in BCS is radiation therapy for local control and systemic biological effect on disease control [30,31].

In a population-based study, the omission of RT after BCS even in the elderly and those who had an increased risk of recurrence was not acceptable because of the increasing potential of local recurrence and breast cancer mortality $[32,33]$. In older patients with low-risk breast cancer, RT in addition to hormonal therapy decreased the probability of local recurrences but did not improve OS [34]. Providing the boost dose of irradiation in external beam radiotherapy (EBRT) and intraoperative radiotherapy (IORT) is another effective issue, with its omission increasing the recurrence to $30 \%$, even in cases with less than $3 \mathrm{~cm}$ tumor size and a negative margin [35]. In another research, the omission of boost dose increased the recurrence rate from 3.6\% to $4.5 \%$ compared with cases who received a boost dose [36]. The reduction of mortality in breast cancer patients, who received IORT as boost or radical dose is another valuable effect of RT in breast cancer patients due to the induced immunological effect of local therapy that reduces cancerrelated death [37-39].

All of our mastectomy cases who were node-positive and had a tumor size of more than five centimeters received RT; also, all BCS cases either received EBRT (boost and whole breast radiation) or full dose/boost of IORT in selected 
patients. This supports the evidence that receiving radiation or not could be considered as an independent variable.

The time of radiation therapy after surgery is another challenging issue, which is not approved in many independent studies [40-43]. In a trial with 16 years follow-up, RT confirmed no increased risk of local recurrence when administered 2, 4, and 7 months after surgery and initial systemic therapy [44]. The radiation dose is also important in OS, for example, treatment with less than $8 \mathrm{GY}$ per week is associated with a breast cancer recurrence rate ranging from $26 \%$ to $30 \%$ [45].

Recently, radiation therapy has been highly considered as a personalized medical treatment because of the molecular markers of each patient and her/his sensitivity to RT; in a study by Kirova, the positive effect of boost was separately confirmed [46].

Many studies have confirmed the immunomodulatory effects of local therapy; radiotherapy promotes the immunological system by inducing immunogenic cell death and subsequently increasing the sensitivity of lymphocytes to tumor cells [37, 47-50]. Studies also have demonstrated that the abscopal effect of local treatment combined with immunotherapy enhances the efficacy of treatment for breast cancer cases in animal models [51].

This study has several limitations. First, regarding its retrospective nature, it is not possible to rule out confounding factors. Moreover, although both groups have received similar adjuvant therapies over time, the changing trend towards BCS in recent years and, thus, the more favorable adjuvant therapies may potentially have had an impact on the overall survival of these patients. Also, we have not investigated the association of biological factors with the survival of patients in each treatment group.

\section{Conclusion}

Our findings are in line with previous studies suggesting an improved survival (OS and DFS) with BCT + RT compared with MRM with/without RT. Thus, BCS should be considered as an appropriate treatment option in breast cancer management when indicated.

\section{Data Availability}

The data used to support the findings of this study are available from the corresponding author upon request.

\section{Conflicts of Interest}

The authors declare that there are no conflicts of interest regarding the publication of this paper.

\section{References}

[1] A. Akbari, M. Khayamzadeh, R. Salmanian et al., "National cancer mortality-to-incidence ratio (MIR) in Iran (2005-2014)," International Journal of Cancer Management, vol. 12, no. 6, Article ID e94145, 2019.

[2] J. H. Breasted, The Edwin Smith Surgical Papyrus, The University of Chicago Press, Chicago, IL USA, 1930.
[3] N. Papavramidou, T. Papavramidis, and T. Demetriou, "Ancient Greek and Greco-Roman methods in modern surgical treatment of cancer," Annals of Surgical Oncology, vol. 17, no. 3, pp. 665-667, 2010.

[4] Avicenna, The Canon of Medicine, Avicenna, The Canon of Medicine, Soroush Publication, Tehran, Iran, 1981.

[5] W. S. Halsted, "The results of operations for the cure of cancer of the breast performed at the johns hopkins hospital from June, 1889, to January, 1894," Annals of Surgery, vol. 20, no. 5, pp. 497-555, 1894.

[6] U. Veronesi, M. Del Vecchio, A. Luini, A. Rasponi, and R. Zucali, "The quadrantectomy, axillary dissection and radiotherapy (QU.A.RT) technique in early breast cancer," International Journal of Surgical Oncology, vol. 6, pp. 141-165, 1983.

[7] U. Veronesi, N. Cascinelli, L. Mariani et al., "Twenty-year follow-up of a randomized study comparing breast-conserving surgery with radical mastectomy for early breast cancer," New England Journal of Medicine, vol. 347, no. 16, pp. 1227-1232, 2002.

[8] U. Veronesi, A. Luini, V. Galimberti, and S. Zurrida, "Conservation approaches for the management of stage I/II carcinoma of the breast: Milan Cancer Institute trials," World Journal of Surgery, vol. 18, no. 1, pp. 70-75, 1994.

[9] B. Fisher, S. Anderson, J. Bryant et al., "Twenty-year follow-up of a randomized trial comparing total mastectomy, lumpectomy, and lumpectomy plus irradiation for the treatment of invasive breast cancer," New England Journal of Medicine, vol. 347, no. 16, pp. 1233-1241, 2002.

[10] B. Fisher, M. Bauer, R. Margolese et al., "Five-year results of a randomized clinical trial comparing total mastectomy and segmental mastectomy with or without radiation in the treatment of breast cancer," New England Journal of Medicine, vol. 312, no. 11, pp. 665-673, 1985.

[11] B. Fisher, C. Redmond, R. Poisson et al., "Eight-year results of a randomized clinical trial comparing total mastectomy and lumpectomy with or without irradiation in the treatment of breast cancer," New England Journal of Medicine, vol. 320, no. 13 , pp. 822-828, 1989.

[12] B. Fisher, S. Anderson, C. K. Redmond, N. Wolmark, D. L. Wickerham, and W. M. Cronin, "Reanalysis and results after 12 years of follow-up in a randomized clinical trial comparing total mastectomy with lumpectomy with or without irradiation in the treatment of breast cancer," New England Journal of Medicine, vol. 333, no. 22, pp. 1456-1461, 1995.

[13] U. Veronesi, B. Salvadori, A. Luini et al., "Breast conservation is a safe method in patients with small cancer of the breast. Long-term results of three randomised trials on 1,973 patients," European Journal of Cancer, vol. 31, no. 10, pp. 1574-1579, 1995.

[14] M. E. Akbari, M. Khayamzadeh, S. J. Khoshnevis, N. Nafisi, and A. Akbari, "Five and ten years survival in breast cancer patients mastectomies vs. Breast conserving surgeries personal experience," International Journal of Cancer Management, vol. 1, no. 2, pp. 53-56, 2008.

[15] R. Sauer, "Adjuvant radiotherapy after breast conserving surgery for breast cancer," European Journal of Cancer, vol. 36, no. 9, pp. 1073-1078, 2000.

[16] O. J. Hartmann-Johnsen, R. Kåresen, E. Schlichting, and J. F. Nygård, "Better survival after breast-conserving therapy compared to mastectomy when axillary node status is positive in early-stage breast cancer: a registry-based follow-up study of 6387 Norwegian women participating in screening, 
primarily operated between 1998 and 2009," World Journal of Surgical Oncology, vol. 15, no. 1, p. 118, 2017.

[17] Y. Sun, L. Mingjuan, H. Liu, and Z. Chenfang, "Comparison of breast-conserving surgery with mastectomy in locally advanced breast cancer after good response to neoadjuvant chemotherapy: a PRISMA-compliant systematic review and meta-analysis," Medicine, vol. 96, no. 43, Article ID e8367, 2017.

[18] A. Nandakumar, G. K. Rath, A. C. Kataki et al., "Decreased survival with mastectomy vis-à-vis breast-conserving surgery in stage II and III breast cancers: a comparative treatment effectiveness study," Journal of Global Oncology, vol. 3, no. 4, pp. 304-313, 2017.

[19] S. Agarwal, L. Pappas, L. Neumayer, K. Kokeny, and J. Agarwal, "Effect of breast conservation therapy vs mastectomy on disease-specific survival for early-stage breast cancer," JAMA Surgery, vol. 149, no. 3, pp. 267-274, 2014.

[20] M. A. Martin, R. Meyricke, T. O’Neill, and S. Roberts, "Breastconserving surgery versus mastectomy for survival from breast cancer: the Western Australian experience," Annals of Surgical Oncology, vol. 14, no. 14, pp. 157-164, 2007.

[21] A. D. Morris, J. F. Wilson, and J. White, "Breast-conserving therapy vs mastectomy in early-stage breast cancer: a metaanalysis of 10-year survival," The cancer journal from Scientific American, vol. 3, no. 1, pp. 6-12, 1997.

[22] M. C. van Maaren, L. de Munck, G. H. de Bock et al., "10 year survival after breast-conserving surgery plus radiotherapy compared with mastectomy in early breast cancer in the Netherlands: a population-based study," The Lancet Oncology, vol. 17 , no. 8, pp. 1158-1170, 2016.

[23] P. Christiansen, S. L. Carstensen, B. Ejlertsen et al., "Breast conserving surgery versus mastectomy: overall and relative survival-a population based study by the Danish Breast Cancer Cooperative Group (DBCG)," Acta Oncologica, vol. 57, no. 1, pp. 19-25, 2018.

[24] M. Lagendijk, M. C. van Maaren, S. Saadatmand et al., "Breast conserving therapy and mastectomy revisited: breast cancerspecific survival and the influence of prognostic factors in 129,692 patients," International Journal of Cancer, vol. 142, no. 1, pp. $165-175,2018$.

[25] B. Fisher, J.-H. Jeong, S. Anderson, J. Bryant, E. R. Fisher, and N. Wolmark, "Twenty-five-year follow-up of a randomized trial comparing radical mastectomy, total mastectomy, and total mastectomy followed by irradiation," New England Journal of Medicine, vol. 347, no. 8, pp. 567-575, 2002.

[26] H. Akbari and M. E. Akbari, "The comparison of three components of breast cancer in females with cancer and healthy ones: coping styles, psychological capital, and patience," Multidisciplinary Cancer Investigation, vol. 2, no. 3, pp. 23-29, 2018.

[27] S. Akbari, F. Dortaj, B. Ranjgar, and M. E. Akbari, "The effect of personality, social support, and life events on overall survival in breast cancer," International Journal of Cancer Management, vol. 12, no. 3, Article ID e83625, 2019.

[28] L. Hoseini, M. E. Akbari, and S. Sarafraz, "Predictive factors influencing the illness perception and quality of life in Iranian breast cancer patients," International Journal of Current Life Sciences, vol. 4, no. 9, pp. 5121-5126, 2014

[29] M. K. Kim, T. Kim, H. G. Moon et al., "Effect of cosmetic outcome on quality of life after breast cancer surgery," $E u$ ropean Journal of Surgical Oncology (EJSO), vol. 41, no. 3, pp. 426-432, 2015.

[30] M. Shahani, B. Arefnejad, H. Zali, N. Nafisi, M. Hashemi et al., "Investigation of IORT-treated tumor bed modification in breast cancer via inducing biological pathways by new comprehensive approaches," in Proceedings of the 10th ISIORT Conference Transcriptomic \& Proteomic, Mannheim, Germany, July 2018.

[31] H. Hakimeh Zali, M. E. Akbari, M. Shahani, J. Shakeri, Sh Jeibouei, and A. Tafti, "Influence of intra-operative radiotherapy (IORT) on tumor bed protein composition of exosome in breast cancer patients," in Proceedings of the 10th ISIORT Conference Transcriptomic \& Proteomic, Mannheim, Germany, July 2018.

[32] T. M. Tuttle, S. Jarosek, E. B. Habermann, D. Yee, J. Yuan, and B. A. Virnig, "Omission of radiation therapy after breastconserving surgery in the United States," Cancer, vol. 118, no. 8, pp. 2004-2013, 2012.

[33] L. Tang, H. Matsushita, and K. Jingu, "Controversial issues in radiotherapy after breast-conserving surgery for early breast cancer in older patients: a systematic review," Journal of Radiation Research, vol. 59, no. 6, pp. 789-793, 2018.

[34] C. Matuschek, B. Edwin, H. Jan et al., "The benefit of adjuvant radiotherapy after breast conserving surgery in older patients with low risk breast cancer-a meta-analysis of randomized trials," Radiation Oncology, vol. 12, no. 1, p. 60, 2017.

[35] M. C. Ryoo, A. R. Kagan, M. Wollin et al., "Prognostic factors for recurrence and cosmesis in 393 patients after radiation therapy for early mammary carcinoma," Radiology, vol. 172, no. 2, pp. 555-559, 1989.

[36] P. Romestaing, Y. Lehingue, C. Carrie et al., "Role of a 10-Gy boost in the conservative treatment of early breast cancer: results of a randomized clinical trial in Lyon, France," Journal of Clinical Oncology, vol. 15, no. 3, pp. 963-968, 1997.

[37] I. Jatoi, J. R. Benson, and I. Kunkler, "Hypothesis: can the abscopal effect explain the impact of adjuvant radiotherapy on breast cancer mortality?" Npj Breast Cancer, vol. 4, no. 1, p. 8, 2018.

[38] A. Salati, M. E. Akbari, N. Nafissi et al., "Comparison of outcome between invasive lobular carcinoma (ILC) and invasive ductal carcinoma (IDC) patients treating with breast conserving surgery (BCS) and radical dose of intraoperative electron radiotherapy (IOERT)," International Journal of Cancer Management, vol. 11, no. 11, Article ID e80985, 2018.

[39] A. Salati, M. E. Akbari, N. Nafissi et al., "Comparing outcome of radical dose intraoperative radiotherapy with electron (IOERT) according to IRIORT consensus and external beam radiotherapy in early breast cancer," Journal of Cancer Science and Therapy, vol. 11, pp. 063-069, 2019.

[40] R. Caponio, P. C. Maria, G. Giusi et al., "Waiting time for radiation therapy after breast-conserving surgery in early breast cancer: a retrospective analysis of local relapse and distant metastases in 615 patients," European Journal of Medical Research, vol. 21, no. 1, p. 32, 2016.

[41] H. K. Koh, K. H. Shin, K. Kim et al., "Effect of time interval between breast-conserving surgery and radiation therapy on outcomes of node-positive breast cancer patients treated with adjuvant doxorubicin/cyclophosphamide followed by taxane," Cancer Research and Treatment, vol. 48, no. 2, pp. 483-490, 2016.

[42] M. C. van Maaren, R. W. Bretveld, J. J. Jobsen et al., "The influence of timing of radiation therapy following breastconserving surgery on 10-year disease-free survival," British Journal of Cancer, vol. 117, no. 2, pp. 179-188, 2017.

[43] V. Barbieri, P. Sanpaolo, and D. Genovesi, "Interval between breast-conserving surgery and start of radiation therapy in early-stage breast cancer is not predictive of local recurrence: a 
single-institution experience," Clinical Breast Cancer, vol. 11, no. 2, pp. 114-120, 2011.

[44] P. Karlsson, B. F. Cole, K. N. Price et al., "Timing of radiation therapy and chemotherapy after breast-conserving surgery for node-positive breast cancer: long-term results from international breast cancer study group trials VI and VII," International Journal of Radiation Oncology $*$ Biology $*$ Physics, vol. 96, no. 2, pp. 273-279, 2016.

[45] J. M. Kurtz, R. Amalric, H. Brandone, Y. Ayme, and J.-M. Spitalier, "How important is adequate radiotherapy for the long-term results of breast-conserving treatment?" $R a$ diotherapy and Oncology, vol. 20, no. 2, pp. 84-90, 1991.

[46] Y. M. Kirova, "Radiation therapy (RT) after breast-conserving surgery (BCS) in 2015-the year of radiation therapy advances," European Journal of Surgical Oncology, vol. 42, no. 4, pp. 437-440, 2016.

[47] N. Brix, A. Tiefenthaller, H. Anders, C. Belka, and K. Lauber, "Abscopal, immunological effects of radiotherapy: narrowing the gap between clinical and preclinical experiences," Immunological Reviews, vol. 280, no. 1, pp. 249-279, 2017.

[48] J. C. Ye and S. C. Formenti, "Integration of radiation and immunotherapy in breast cancer-treatment implications," The Breast, vol. 38, pp. 66-74, 2018.

[49] Z. I. Hu, H. L. McArthur, and A. Y. Ho, "The abscopal effect of radiation therapy: what is it and how can we use it in breast cancer?" Current Breast Cancer Reports, vol. 9, no. 1, pp. 45-51, 2017.

[50] A. Azami, N Suzuki, Y Azami et al., "Abscopal effect following radiation monotherapy in breast cancer: a case report," Molecular and Clinical Oncology, vol. 9, no. 9, pp. 283-286, 2018.

[51] M. E. Rodriguez-Ruiz, R. Inmaculada, M. Lina et al., “TGF $\beta$ blockade enhances radiotherapy abscopal efficacy effects in combination with anti-PD1 and anti-CD137 immunostimulatory monoclonal antibodies," Molecular Cancer Therapeutics, vol. 18, 2019. 